Kompass

Ophthalmologie

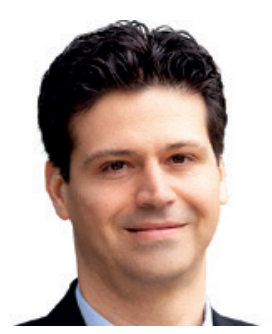

Stylianos Michalakis

Augenklinik und Poliklinik des Klinikums der Universität München, München, Deutschland

\title{
Gentherapie bei erblichen Netzhautdystrophien
}

Beeinträchtigungen des Sehvermögens haben schwerwiegende Auswirkungen auf das tägliche Leben, die sozialen Interaktionen und sogar auf die psychische Gesundheit der betroffenen Personen. Zwar gibt es viele verschiedene erworbene Erkrankungen, die zur Beeinträchtigung des Sehvermögens führen, doch gehören die erblichen Netzhautdystrophien zu den größten Herausforderungen, wenn es um therapeutische Maßnahmen geht. Erbliche Netzhauterkrankungen werden durch pathogene Varianten in einem der mehr als 250 bekannten Krankheitsgenen verursacht. Je nach betroffenem Gen können sie sich klinisch unterschiedlich äußern und von einer leichten Seheinschränkung bis zur völligen Erblindung führen. Während einzelne erbliche Netzhauterkrankungen selten sind, liegt die Gesamtprävalenz bei 1:2000, womit sie die Hauptursache für Sehkraftverlust in der Bevölkerung im erwerbstätigen Alter sind [1].

Bis vor kurzem gab es keine Behandlungsmöglichkeiten, die die mutationsbedingten Funktionsstörungen ausgleichen konnten. Dies hat sich mit der Verfügbarkeit wirksamer und sicherer viraler Genvektoren geändert, was dazu geführt hat, dass die Gentherapie zu einer validen Option für die Behandlung erblicher Netzhauterkrankungen geworden ist. Einige der vielversprechendsten Behandlungskonzepte sind genspezifische Ansätze, die auf rekombinanten Adeno-assoziierten Viren (AAV) beruhen. Die Prävalenz von AAVs ist in der menschlichen
Bevölkerung sehr hoch; eine Infektion mit diesem Virus wurde jedoch bisher mit keiner klinischen Pathologie oder Krankheit beim Menschen in Verbindung gebracht. Zudem zeigen AAV-Vektoren eine geringe, klar Dosis-abhängige Toxizität und Immunogenität. AAVs sind zwar nicht-integrierende Viren, aber das AAV-Vektorgenom persistiert als Episom im Kern der Zielzellen und sorgt für eine stabile und langfristige Genexpression in sich nicht teilenden Zellen, wie z.B. dem retinalen Pigmentepithel. Diese positiven Eigenschaften in Verbindung mit hoher Effizienz und breitem Tropismus haben AAV-Vektoren zur aktuell vielversprechendsten Vektortechnologie werden lassen.

Voretigene neparvovec (Luxturna ${ }^{\circledR}$ ) ist die erste und bisher einzige zugelassene AAVbasierte Gentherapie zur Behandlung der RPE65-bedingten Netzhautdystrophie [2]. Voretigene neparvovec nutzt das Behandlungskonzept der Gensupplementierungstherapie (auch Gen-Augmentation genannt). Dieses Konzept ist im Prinzip auf alle autosomal rezessiv vererbten Netzhauterkrankungen anwendbar und sieht vor, dass mit Hilfe des AAV-Vektors eine gesunde Kopie des beim Patienten mutierten Gens (in diesem Fall RPE65) in die betroffenen Zellen der Netzhaut eingebracht wird. Ziel ist es, eine fehlende Genfunktion in den betroffenen Netzhautzellen eines Patienten zu ergänzen, um einen funktionellen Nutzen und längerfristig eine Aufrechterhaltung der Netzhautmorphologie zu erreichen. 
Voretigene neparvovec wird als einmalige Therapie lokal durch subretinale Injektion unter die Netzhaut verabreicht, um die Oberfläche der Zielzellen (das retinale Pigmentepithel) zu erreichen, sie zu transduzieren und dort die Langzeitexpression des RPE65-Gens zu induzieren.

Bisher wurden Hunderte von RPE65-Varianten identifiziert, von denen einige als pathogen oder wahrscheinlich pathogen eingestuft wurden, während die Bedeutung vieler anderer noch nicht geklärt ist. Der Übersichtsartikel gibt einen Überblick über die genetische Diagnostik, die erforderlich ist, um Patienten auszuwählen, die für eine Behandlung mit Voretigene neparvovec in Frage kommen [2]. Der Anwendungsbericht fasst die Behandlungsergebnisse von fünf Patienten mit bi-allelischen RPE65-Mutationen zusammen, die mit Voretigene Neparvovec behandelt wurden [3]. In der Arbeit werden Messprotokolle vorgestellt, um die räumlichen und zeitlichen Auswirkungen der Gentherapie zu bewerten, funktionelle Veränderungen in retinotopischen Karten der Stäbchen- und Zapfenfunktion zu messen und somit komplementäre Aspekte der Netzhautfunktion zu beurteilen.

\section{Literatur}

1 Hanany M, Rivolta C, Sharon D: Worldwide carrier frequency and genetic prevalence of autosomal recessive inherited retinal diseases. Proceedings of the National Academy of Sciences of the United States of America 2020; 117:2710-2716.

2 Aoun M, Passerini I, Chiurazzi P, et al.: Inherited Retinal Diseases Due to RPE65 Variants: From Genetic Diagnostic Management to Therapy. Int J Mol Sci 2021;22(13):7207.

3 Stingl K, Kempf M, Bartz-Schmidt KU, et al.: Spatial and temporal resolution of the photoreceptors rescue dynamics after treatment with voretigene neparvovec. $\mathrm{Br} \mathrm{J}$ Ophthalmol 2021;DOI:10.1136/bjophthalmol-2020318286. 